\title{
Hipertensão secundária: abordagem nos cuidados de saúde primários
}

Tiago Francisco da Cunha Costaํ, Diana Catarina Coelho Bernardo Leitão

\section{RESUMO}

Objetivos: Identificar situações sugestivas de hipertensão secundária (HS). Rever a fisiopatologia, manifestações clínicas e/ou laboratoriais, diagnóstico e tratamento das principais formas de HS, bem como estabelecer o papel do médico de família na sua abordagem à luz da evidência mais recente.

Fontes de dados: MEDLINE/PubMed, The Cochrane Library, UpToDate, U.S. Preventive Services Task Force e normas de orientação clínica da Direção-Geral da Saúde.

Métodos: Foi realizada a pesquisa de artigos científicos em plataformas online de medicina baseada em evidência utilizando as palavras-chave Secondary Hypertension e Primary Health Care. A pesquisa foi realizada em julho de 2019, sendo a seleção dos artigos feita com base no título, conteúdo do resumo e data de publicação. Foram também consultadas normas de orientação clínica e guidelines.

Resultados: A HS deve ser suspeita em contexto de hipertensão resistente, início de hipertensão antes dos 30 anos num utente sem fatores de risco, elevação súbita ou instabilidade da pressão arterial basal, hipertensão maligna ou acelerada, início de hipertensão diastólica em idosos, hipertensão associada a distúrbios hidro-eletrolíticos ou hipertensão associada a clínica sugestiva de determinada etiologia específica. Em crianças e adolescentes, as principais causas de HS são as doenças do parênquima renal, ao passo que nos adultos as etiologias mais comuns incluem a síndroma da apneia obstrutiva do sono, doenças do parênquima renal, estenose da artéria renal, hiperaldosteronismo primário e o feocromocitoma. O reconhecimento de manifestações clínicas e/ou laboratoriais sugestivas da etiologia da HS deve orientar a realização dos testes de rastreio para posterior tratamento ou referenciação.

Conclusão: Avaliar em todos os utentes hipertensos a existência de causas secundárias não é custo-efetivo, pelo que apenas devem ser estudados os utentes com características sugestivas de HS. A realização e interpretação dos testes de rastreio de forma adequada é fundamental para a referenciação e tratamento dos utentes com HS.

Palavras-chave: Hipertensão secundária; Cuidados de saúde primários; Apneia obstrutiva do sono; Doenças do parênquima renal; Hipertensão renovascular; Hiperaldosteronismo primário.

\section{INTRODUÇÃO}

A hipertensão secundária (HS) é a hipertensão devida a uma causa identificável, que pode ser tratada com uma intervenção dirigida à condição subjacente. ${ }^{1}$

Estima-se que 5 a $10 \%$ dos hipertensos apresentem HS; ${ }^{1-3}$ no entanto, esta prevalência pode variar consoante a faixa etária. ${ }^{1-2}$ Em crianças com menos de 12

1. Médico Interno de Medicina Geral e Familiar. USF Hygeia, ACeS Tâmega III - Vale do Sousa Norte. Lixa, Portugal.

2. Mestrado Integrado em Medicina. Instituto de Ciências Biomédicas Abel Salazar. Porto, Portugal. anos de idade, 70 a $85 \%$ dos casos de hipertensão são devidos a causas secundárias, diminuindo a sua prevalência a partir desta idade. ${ }^{1}$

As etiologias mais frequentes de HS são igualmente variáveis de acordo com os diferentes grupos etários (Tabela 1).,

É comum a hipertensão primária coexistir com causas secundárias de hipertensão, ${ }^{2}$ o que pode explicar o facto de a hipertensão poder não ser completamente revertida após a resolução da condição secundária subjacente. ${ }^{2,4-5}$ Por outro lado, a HS de longa duração, ao promover um remodelling vascular irreversível, pode 
contribuir para a manutenção de hipertensão residual após a remoção da causa. ${ }^{2,6}$ De modo a minimizar este efeito, a deteção e o tratamento precoce são fundamentais.

Em Portugal está recomendada a medição da pressão arterial a partir dos três anos de idade, apesar de a evidência atual ser insuficiente para avaliar os riscos/benefícios deste rastreio..$^{7-8} \mathrm{Um}$ dos objetivos deste início precoce da avaliação é a deteção de casos de HS. ${ }^{8}$

TABELA 1. Principais causas de hipertensão secundária de acordo com a faixa etária
\begin{tabular}{l|l} 
Faixa etária & \multicolumn{1}{c|}{ Causas mais comuns } \\
\hline Crianças e adolescentes ( $\leq 18$ anos) & - Doenças do parênquima renal \\
& - Coartação da aorta \\
\hline Adultos jovens (19-39 anos) & - Doenças do parênquima renal \\
& - Estenose da artéria renal por displasia fibromuscular \\
& - Disfunção tiroideia \\
\hline Adultos de meia-idade (40-64 anos) & - Hiperaldosteronismo primário \\
& - Síndroma da apneia obstrutiva do sono \\
& - Síndroma de Cushing \\
\hline Adultos idosos ( $\geq 65$ anos) & - Deocromocitoma \\
\hline & - Estenças do parênquima renal da artéria renal aterosclerótica \\
& - Disfunção tiroideia \\
\hline
\end{tabular}

Como a maioria dos hipertensos é abordada ao nível dos cuidados de saúde primários (CSP), ${ }^{6}$ é importante que os médicos de família estejam familiarizados com a identificação de situações sugestivas de HS, de modo a orientar os utentes adequadamente e reduzir a sua morbimortalidade. Por outro lado, estas causas de HS são frequentemente alvo de atualização nos seus testes de rastreio/diagnóstico, pelo que importa a sua constante revisão.

Deste modo, esta revisão tem como objetivo reunir a evidência mais recente sobre as causas de HS, nomeadamente a sua fisiopatologia, manifestações clínicas e/ou laboratoriais, diagnóstico e tratamento, bem como estabelecer o papel do médico de família na sua abordagem.

\section{MÉTODOS}

Numa primeira fase foi realizada a pesquisa de artigos de revisão simples e sistemática em plataformas online de medicina baseada na evidência, nomeadamente na PubMed, The Cochrane Librarye UpToDate, utilizando as palavras-chave Secondary Hypertension e Primary Health Care. Numa segunda fase, de forma a obter informação adicional sobre cada uma das causas secundárias identificadas, foi feita uma nova pesquisa de artigos de revisão, ensaios clínicos aleatorizados e normas de orientação clínica através das palavras-chave Obstructive sleep apnea, Renal parenchymal diseases,
Renovascular hypertension, Primary hyperaldosteronism, Thyroid dysfunction, Cushing syndrome, Paraganglioma, Pheochromocytoma e Aortic coarctation.

Ambas as pesquisas foram efetuadas em julho de 2019, tendo as mesmas sido limitadas a artigos publicados a partir do ano de 2000.

Os artigos foram incluídos com base no título, conteúdo do resumo, relevância, idioma de publicação (artigos em língua inglesa e portuguesa) e data de publicação. Por sua vez, foram excluídos artigos cuja evidência não fosse a mais recente à data da pesquisa. A recuperação manual de artigos relevantes da lista de referências bibliográficas dos artigos selecionados foi também realizada.

Esta revisão, pela sua abrangência ao nível das patologias abordadas, poderá apresentar um potencial risco de viés de seleção dos artigos. Procurou-se minimizar este risco com a seleção dos artigos após a concordância de ambos os autores.

\section{RESULTADOS}

1. Características sugestivas de hipertensão secundária

Uma vez que não é custo-efetivo avaliar em todos os utentes hipertensos a existência de causas secundárias para a hipertensão, apenas devem ser estudados os utentes com características sugestivas, ${ }^{1-4,9-10}$ descritas na Tabela 2. 
TABELA 2. Características sugestivas de hipertensão secundária

Hipertensão resistente (pressão arterial acima dos valores-alvo, apesar do uso de três classes de fármacos anti-hipertensores em doses otimizadas, incluindo um diurético)

Elevação súbita ou instabilidade na pressão arterial basal

Início de hipertensão antes dos 30 anos num utente não obeso, não negro e sem história familiar de hipertensão

Anamnese e/ou exame objetivo sugestivos de determinada etiologia específica com associação à hipertensão

Hipertensão maligna ou acelerada (e.g., utentes com hipertensão grave e sinais de lesão de órgão-alvo, como hemorragia

retiniana ou papiledema, insuficiência cardíaca, alterações neurológicas ou lesão renal aguda)

Início de hipertensão diastólica em idosos ( $\geq 65$ anos)

Lesões de órgão-alvo desproporcionais ao grau de hipertensão

Hipertensão associada a distúrbios hidroeletrolíticos, como hipocalémia e alcalose metabólica

Idade comprovada de início da hipertensão antes da puberdade

Utentes não-dipper

Perante estes achados, e após exclusão de incumprimento terapêutico, hipertensão de bata branca e pseudo-hipertensão, esta última sobretudo no doente idoso, deve ser realizado um estudo dirigido à causa suspeita. ${ }^{9,11}$ A extensão da avaliação de causas secundárias deve sempre depender do estado geral do utente, probabilidade de diagnóstico e ponderação dos riscos do tratamento/falta de diagnóstico. ${ }^{4}$

\section{Principais causas de hipertensão secundária \\ 2.1. Síndroma da apneia obstrutiva do sono}

A síndroma da apneia obstrutiva do sono (SAOS) é uma condição caracterizada por episódios recorrentes de apneias e hipopneias, causados pelo colapso das vias aéreas superiores durante o sono. ${ }^{12} \mathrm{~A}$ sua prevalência é de cerca de $2-4 \%$ nos homens e $1-2 \%$ nas mulheres de meia-idade. ${ }^{13}$ Estima-se que cerca de 70 a $90 \%$ dos utentes com hipertensão resistente (HR) apresentem SAOS. ${ }^{14-15}$

\subsubsection{Fisiopatologia}

Na SAOS o colapso das vias aéreas provoca hipóxia e hipercapnia, que são estimuladores da atividade simpática. ${ }^{15-16} \mathrm{~A}$ atividade simpática leva a fenómenos de vasoconstrição e à ativação do sistema renina-angiotensina-aldosterona (SRAA), com consequente aumento da pressão arterial. ${ }^{17} \mathrm{~A}$ fragmentação do sono também se associa a aumento da pressão arterial, frequência cardíaca e stress oxidativo. ${ }^{16}$

\subsubsection{Manifestações clínicas e/ou laboratoriais}

A SAOS é mais comum em indivíduos do sexo masculino, com idade superior a 50 anos e que apresentem roncopatia ou aumento da circunferência cervical. ${ }^{10,18}$

Apesar de a SAOS cursar frequentemente com hipersonolência diurna, vários estudos mostraram que apenas uma minoria dos utentes com HR e SAOS apresenta esta manifestação, ${ }^{15,18-20}$ o que pode estar associado à ativação crónica do sistema nervoso simpático. ${ }^{18}$ Desta forma, a hipersonolência diurna não parece ser um fator preditivo para SAOS nos utentes com HR. ${ }^{18}$

Outras manifestações incluem cefaleias matinais e alteração das funções cognitivas, como a concentração e memória, irritabilidade, depressão e ansiedade. ${ }^{2,16}$

\subsubsection{Diagnóstico}

$\mathrm{Na}$ abordagem inicial do doente com suspeita de SAOS podem ser aplicados questionários que auxiliam no seu rastreio, como o Questionário de Berlim e a Escala de Sonolência de Epworth. ${ }^{16,18}$ No entanto, o diagnóstico definitivo requer a realização de uma polissonografia, que permite também classificar a SAOS consoante a sua gravidade: ligeira [Índice de apneias-hipopneias (IAH) entre 5 e 15], moderada (IAH entre 16-30) ou grave (IAH superior a 30 ). ${ }^{12,21}$

É recomendado que utentes com SAOS e hipertensão realizem um ecocardiograma, de forma a avaliar as dimensões e espessura das cavidades, função 
sistólica ventricular e estimar a pressão da artéria pulmonar. $^{2}$

\subsubsection{Tratamento}

A SAOS foi identificada como a causa secundária de hipertensão mais passível de ser tratada. ${ }^{15}$

O tratamento mais consensual é a utilização de CPAP (Continuous Positive Airway Pressure). ${ }^{14-16} \mathrm{O}$ início de prescrição do CPAP deve ser efetuado em centros de referência de medicina do sono, idealmente que integrem pneumologistas, otorrinolaringologistas, neurologistas e internistas. ${ }^{22}$ Globalmente, o tratamento com CPAP associa-se a melhor controlo da pressão arterial, qualidade do sono e diminuição da mortalidade. ${ }^{21}$

O uso de um antagonista dos recetores dos mineralocorticoides, como a espironolactona, pode reduzir significativamente o IAH, a dessaturação noturna e a pressão arterial, constituindo uma alternativa terapêutica. ${ }^{14,23}$ A adoção de um estilo de vida saudável traz benefícios adicionais no controlo da SAOS e da hipertensão. ${ }^{6}$

\subsection{Doenças do parênquima renal}

As doenças do parênquima renal (DPR) incluem as doenças glomerulares, as doenças tubulo-intersticiais, a doença renal poliquística e a nefropatia diabética. ${ }^{6} \mathrm{As}$ DPR são a causa mais comum de hipertensão em crianças. ${ }^{3}$ Estima-se que a sua prevalência a nível mundial esteja próxima dos $9,6 \%,{ }^{24}$ estando presente em aproximadamente 2 a $10 \%$ dos utentes com HR. ${ }^{2}$

Com o envelhecimento da população é expectável que o número de utentes com DPR aumente e, consequentemente, o número de casos de HS. ${ }^{25}$

\subsubsection{Fisiopatologia}

A hipertensão surge como consequência da progressão da doença renal, em virtude do menor número de nefrónios disponíveis para filtração de água e sódio. Esta diminuição da função renal também leva a um aumento da atividade do sistema nervoso simpático e à menor eficácia dos fármacos anti-hipertensores. ${ }^{14}$

\subsubsection{Manifestações clínicas e/ou laboratoriais}

As manifestações clínicas das DPR dependem da etiologia subjacente e do grau de atingimento da função renal. Importa salientar que o rim é um órgão capaz de se adaptar à perda progressiva da sua função, pelo que muitas das manifestações clínicas típicas apenas surgem quando a função renal se encontra significativamente deteriorada. ${ }^{26}$

A poliúria pode ser uma manifestação precoce de DPR, assim como a elevação da creatinina sérica. Outras manifestações incluem alterações das características macroscópicas da urina (cor e aspeto), proteinúria, edemas, oligoanúria, fadiga, anorexia, náuseas e vómitos. ${ }^{26} \mathrm{Em}$ crianças, as DPR podem manifestar-se através de um atraso de crescimento. ${ }^{27}$

\subsubsection{Diagnóstico}

As DPR devem ser suspeitadas perante elevação da creatinina e ureia plasmática e/ou evidência de proteinúria, hematúria ou cilindros na urina. ${ }^{3,10,28} \mathrm{~A}$ ecografia renal permite avaliar sinais de cronicidade e pode fazer o diagnóstico de algumas etiologias de DPR, sendo o exame de primeira linha para investigação etiológica em crianças. ${ }^{3}$

A investigação adicional das DPR deve ser realizada por um nefrologista, pelo que se recomenda a referenciação do utente. ${ }^{6}$

\subsubsection{Tratamento}

A abordagem terapêutica da hipertensão associada às DPR deve ter por objetivo, para além da redução da pressão arterial, reduzir o risco cardiovascular e atrasar a progressão da doença renal. ${ }^{28} \mathrm{O}$ tratamento pode variar consoante a etiologia específica da DPR. No entanto, para a hipertensão estão recomendadas medidas dietéticas, tratamento farmacológico com um inibidor da enzima conversora da angiotensina (IECA) ou antagonista dos recetores da angiotensina (ARA) e um diurético, ou diálise em casos mais avançados. ${ }^{14,28-29}$

Os IECA/ARA estão recomendados particularmente se existir albuminúria, uma vez que reduzem a pressão intraglomerular e abrandam o declínio da função renal. ${ }^{30}$ Elevações inferiores a $30 \%$ na creatinina plasmática são aceitáveis após a introdução do IECA/ARA. ${ }^{28}$

Os diuréticos recomendados são os diuréticos de ansa, devendo ser administrados até ser atingida a pressão arterial alvo ou o "peso seco». ${ }^{29}$

\subsection{Estenose da artéria renal}

A hipertensão causada por estenose da artéria renal (EAR), também designada por hipertensão reno-vascular, 
é uma das causas de HS com maior potencial de reversibilidade..$^{10} \mathrm{~A}$ EAR está presente em cerca 1 a $8 \%$ da população hipertensa e em 2,5 a 20\% da população com HR. ${ }^{5}$

Em adultos jovens, especialmente do sexo feminino, a causa mais comum de EAR é a displasia fibromuscular, ao passo que na população idosa predomina a doença aterosclerótica. ${ }^{3,31} \mathrm{~A}$ EAR está frequentemente presente em indivíduos com doença arterial aterosclerótica. ${ }^{32}$ No entanto, o rastreio da EAR em utentes com doença aterosclerótica que não apresentem HR ou função renal anormal não está recomendado. ${ }^{2-3}$

\subsubsection{Fisiopatologia}

A EAR pode ser causada por doença aterosclerótica, displasia fibromuscular, arterite ou lesão ocupante de espaço com compressão extrínseca da artéria renal. ${ }^{6} \mathrm{~A}$ diminuição do lúmen vascular condiciona hipoperfusão e isquemia renal, estimulando a libertação de renina pelas células justaglomerulares renais. ${ }^{33} \mathrm{~A}$ libertação de renina ativa o SRAA, elevando a pressão arterial. ${ }^{33}$

\subsubsection{Manifestações clínicas e/ou laboratoriais}

A EAR possui um amplo espetro de manifestações clínicas, que vão desde um achado incidental e assintomático até a um estado de hipertensão acelerada ou insuficiência renal. ${ }^{14}$ No entanto, existem algumas manifestações particularmente sugestivas, que incluem:

- Aumento agudo de mais de 50\% nos níveis de creatinina sérica após introdução de um IECA/ARA; ;,10

- Hipertensão num utente que apresenta uma diferença no tamanho dos rins superior a $1,5 \mathrm{~cm} ; 3,6,10$

- Hipertensão grave em mulheres de idade jovem ou em utentes mais velhos com doença aterosclerótica conhecida; ${ }^{3,10,28}$

- Utentes com insuficiência cardíaca com fração de ejeção preservada e congestão pulmonar recorrente; ${ }^{34}$

- Auscultação de um sopro abdominal (com sensibilidade de $40 \%$ e especificidade de $99 \%$ ). ${ }^{3}$

\subsubsection{Diagnóstico}

Perante a suspeita de EAR, o exame de primeira linha é a ecografia com doppler das artérias renais, sendo um exame de rastreio eficaz quando realizado por um operador experiente. ${ }^{35} \mathrm{~A}$ realização de angiografia por TC (tomografia computorizada) ou RM (ressonância magnética), apesar da maior sensibilidade e espe- cificidade, é limitada pelo elevado custo e a possível falta de acessibilidade nos CSP. ${ }^{36}$

A angiografia da artéria renal é o gold-standard para diagnóstico de EAR, devendo apenas ser realizada em ambiente hospitalar, caso seja considerada a realização de intervenção cirúrgica ou percutânea pela cirurgia vascular. ${ }^{3,5-6}$

\subsubsection{Tratamento}

O tratamento recomendado para a maioria dos utentes com EAR é o controlo da pressão arterial com IECA/ARA. ${ }^{37}$ Estes fármacos são bem tolerados pela maioria dos utentes; no entanto, devem ser iniciados em baixa dose, monitorizando a função renal uma ou duas semanas após início da terapêutica. ${ }^{14,37}$

Utentes que apresentem displasia fibromuscular, deterioração da função renal, hipertensão refratária ou congestão pulmonar de repetição são candidatos a cirurgia ou intervenção percutânea de revascularização da artéria renal. ${ }^{38-39}$

\subsection{Hiperaldosteronismo primário}

O hiperaldosteronismo primário (HP) é um grupo de doenças cuja produção de aldosterona é inapropriadamente elevada para o nível de sódio plasmático, autónoma relativamente aos seus mecanismos de regulação e não suprimível pela ingestão de sódio. ${ }^{40} \mathrm{Nas}$ suas formas mais comuns, o excesso de secreção de aldosterona é devido a um adenoma ou à hiperplasia do córtex das glândulas suprarrenais. ${ }^{41}$

O HP tem vindo a ser reconhecido cada vez mais como uma causa corrigível de HR, sendo estimado em 10 a $20 \%$ dos utentes com HS. ${ }^{23}$

\subsubsection{Fisiopatologia}

A produção inapropriadamente elevada de aldosterona promove uma absorção excessiva de sódio, causando a expansão do volume intravascular. ${ }^{42} \mathrm{~A}$ aldosterona é também responsável pelo desenvolvimento de rigidez arterial, disfunção endotelial e hipertrofia/fibrose ventricular esquerda, que contribuem para elevar a pressão arterial. ${ }^{14,42}$

\subsubsection{Manifestações clínicas e/ou laboratoriais}

Dever-se-á suspeitar da existência de HP como causa da hipertensão num utente com hipocalémia inex- 


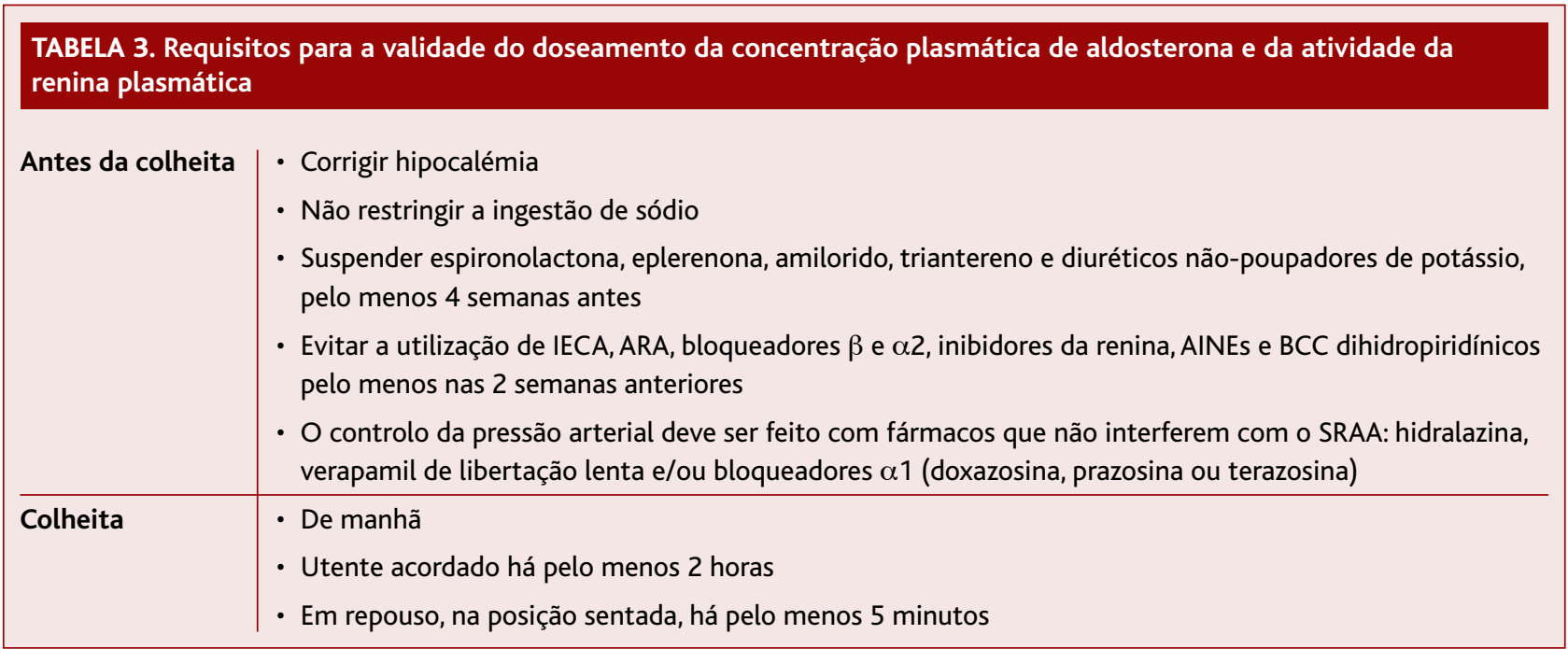

Legenda: AINEs = Anti-inflamatórios não esteroides; ARA = Antagonistas dos recetores da angiotensina; BCC = Bloqueadores dos canais de cálcio; IECA = Inibidores da enzima conversora da angiotensina; SRAA = Sistema renina-angiotensina-aldosterona.

plicável ou induzida por diuréticos, identificação de um nódulo na glândula suprarrenal ou familiares de $1^{\circ}$ grau com HP. ${ }^{40}$ É importante referir que, apesar da hipocalémia ser uma manifestação clínica muito sugestiva de HP, esta não está presente no momento do diagnóstico de HP em mais de $50 \%$ dos casos. ${ }^{4,10,31}$ Assim, está recomendado rastrear HP em todos os utentes com suspeita de HS. ${ }^{40}$

\subsubsection{Diagnóstico}

De acordo com as guidelines da Endocrine Society, ${ }^{40}$ o diagnóstico de HP envolve três etapas sequenciais: rastreio, testes confirmatórios e diferenciação do subtipo. Uma vez que estas duas últimas etapas do diagnóstico são mais complexas, alguns autores defendem que os utentes devem ser referenciados a um endocrinologista após um teste de rastreio positivo. ${ }^{14}$ Estudos de imagem da suprarrenal devem ser protelados até ser confirmada a produção inadequada de aldosterona. ${ }^{4}$

O teste de rastreio do HP mais consensual é a medição da razão entre a concentração plasmática de aldosterona e a atividade da renina plasmática. ${ }^{10,14,40-42} \mathrm{O}$ teste é considerado positivo se essa razão for superior a $30(\mathrm{ng} / \mathrm{dL}$ por $\mathrm{ng} / \mathrm{mL} / \mathrm{h}),{ }^{40}$ podendo variar consoante o laboratório. ${ }^{40,43}$

Para o teste de rastreio ter validade é fundamental uma preparação adequada (Tabela 3). Se o teste de ras- treio for inconclusivo ou negativo, mas existir uma elevada suspeita clínica, este deve ser repetido. ${ }^{40}$

\subsubsection{Tratamento}

Nos utentes com doença unilateral, quer por adenoma ou hiperplasia, a adrenalectomia laparoscópica unilateral apresenta resultados muito satisfatórios. No entanto, se os utentes não apresentarem critérios para cirurgia ou rejeitarem essa opção, deve ser proposto tratamento médico com um antagonista dos recetores dos mineralocorticoides. ${ }^{40,42}$

Nos utentes com doença bilateral está recomendado o tratamento médico, sendo a espironolactona o fármaco de $1^{\mathrm{a}}$ linha. ${ }^{40,42}$

\section{Causas menos comuns de hipertensão secundária 3.1. Disfunção tiroideia}

A disfunção da glândula tiroideia, tanto em estados de hipertiroidismo/tireotoxicose como de hipotiroidismo, tem sido associada a hipertensão. ${ }^{44} \mathrm{~A}$ prevalência estimada de disfunção tiroideia nos utentes hipertensos é de 1 a $2 \%$, sendo 1 a $3 \%$ nos utentes com HR. ${ }^{2}$

\subsubsection{Fisiopatologia}

No hipotiroidismo, a diminuição do débito cardíaco causa um aumento compensatório da resistência 
vascular periférica com o objetivo de manter uma perfusão adequada dos tecidos, o que conduz a hipertensão, sobretudo, diastólica. ${ }^{45}$ No hipertiroidismo verifica-se um aumento da frequência, contratilidade e débito cardíaco, aumentando predominantemente a pressão arterial sistólica. ${ }^{45-46}$

\subsubsection{Manifestações clínicas e/ou laboratoriais}

As manifestações clínicas associadas ao hipertiroidismo são semelhantes a um estado hiperadrenérgico, podendo surgir palpitações, tremores, insónia, perda de peso, aceleração do trânsito intestinal, intolerância ao calor ou hiperatividade. Ao exame físico são achados sugestivos de hipertiroidismo a presença de exoftalmia, taquicardia e/ou aumento da pressão de pulso. ${ }^{5}$

Em contrapartida, sintomas como letargia, obstipação, intolerância ao frio, aumento de peso e mixedema sugerem hipotiroidismo. Bradicardia e estreitamento da pressão de pulso são sinais frequentemente presentes. ${ }^{44}$

\subsubsection{Diagnóstico}

O doseamento da hormona estimulante da tiroide (TSH) é o melhor teste de rastreio da disfunção tiroideia. ${ }^{2-3}$ Em caso de alterações, deve ser realizada uma nova avaliação com o doseamento da TSH e T4 livre, de modo a caracterizar melhor a disfunção em causa. ${ }^{45,47}$

\subsubsection{Tratamento}

O tratamento da hipertensão associada às disfunções tiroideias habitualmente só se consegue após ser alcançado o estado de eutiroidismo. ${ }^{45}$

O tratamento do hipertiroidismo envolve a diminuição da síntese/libertação das hormonas tiroideias, com recurso a tratamento médico ou cirúrgico, e o controlo de sintomas com bloqueadores $\beta{ }^{44,47}$ Os utentes devem ser referenciados a um endocrinologista. ${ }^{48}$

No hipotiroidismo, a base do tratamento é a reposição das hormonas tiroideias com levotiroxina. ${ }^{5,44}$

\subsection{Síndroma de Cushing}

A síndroma de Cushing (SC) resulta do excesso de glicocorticoides no organismo. A principal causa de SC é a administração exógena de glicocorticoides (hipercortisolismo exógeno). ${ }^{44-45}$

O aumento da produção endógena (hipercortisolismo endógeno) é uma causa rara, com uma prevalência de 1,4 a 10 casos por milhão de habitantes. ${ }^{5}$ A maioria destes casos resulta de um adenoma hipofisário produtor de ACTH, sendo esta condição conhecida como doença de Cushing. ${ }^{49}$ Outras causas menos comuns incluem a produção ectópica de ACTH e o adenoma/carcinoma/hiperplasia nodular da suprarrenal. ${ }^{44}$

A hipertensão pode ser encontrada em até $93 \%$ dos casos de SC. ${ }^{50}$

\subsubsection{Fisiopatologia}

O hipercortisolismo pode desencadear hipertensão por vários mecanismos, como o aumento da atividade do sistema nervoso simpático, maior sensibilidade aos agonistas adrenérgicos, menor destruição das células produtoras de catecolaminas e ativação do SRAA e da endotelina- $1 .{ }^{1-52}$

\subsubsection{Manifestações clínicas e/ou laboratoriais}

Devemos suspeitar da SC como causa de HS perante um utente que apresente um fácies em «lua cheia» (fácies cushingoide), fragilidade cutânea, equimoses fáceis, obesidade abdominal centrípeta, adiposidade dorso-cervical («pescoço de búfalo»), osteoporose inexplicável, atraso no crescimento, fraqueza muscular proximal, estrias roxo-avermelhadas, perfil de pressão arterial não-dipper, perturbação do humor, hirsutismo, acne, alopecia, irregularidades menstruais, suscetibilidade aumentada a infeções ou diminuição da tolerância à glicose. . $^{10,4-45,51}$ Note-se que em até $15 \%$ dos adultos com SC, as manifestações clínicas podem ocorrer apenas periodicamente. ${ }^{53}$

A hipótese de SC também deve ser considerada perante um utente que apresente um incidentaloma na suprarrenal, mesmo na ausência de manifestações clínicas. ${ }^{54}$

\subsubsection{Diagnóstico}

Utentes com suspeita de HS, mas que não apresentem características sugestivas de SC, não necessitam de ser estudados quanto à sua presença. ${ }^{10}$ No entanto, o diagnóstico de SC pode ser difícil, mesmo naqueles que apresentam manifestações clínicas, pelo facto de este compartilhar muitas características com a síndroma metabólica. ${ }^{52}$

A abordagem diagnóstica nos CSP de um utente com suspeita de SC encontra-se esquematizada na Figura $1 .{ }^{45,55-57}$ 


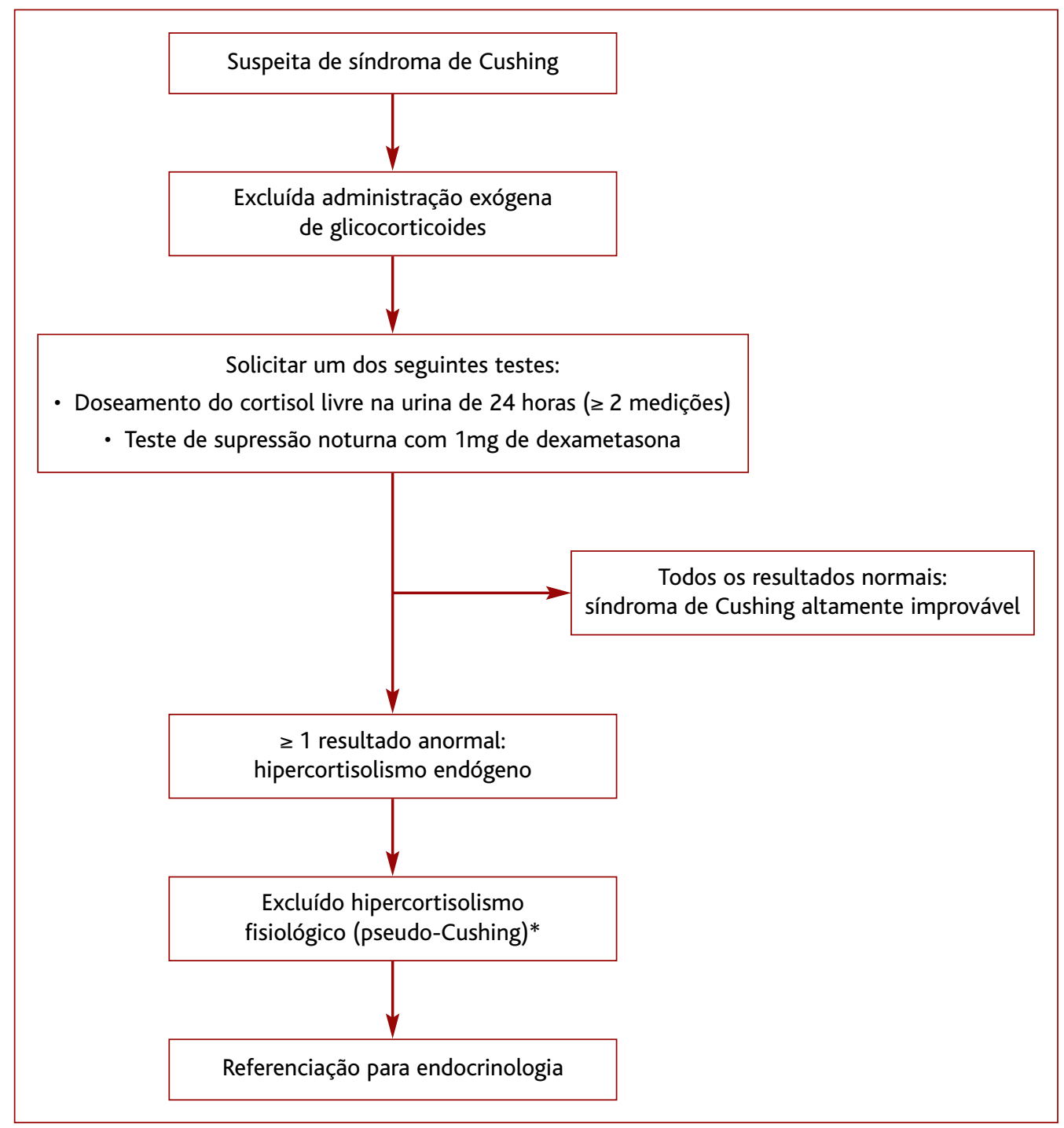

Figura 1. Algoritmo diagnóstico para utentes com suspeita de síndroma de Cushing.

*Causas de Pseudo-Cushing: obesidade severa, gravidez, depressão, ansiedade, stress físico (hospitalizações, dor), exercício vigoroso, trabalho por turnos, alcoolismo ou o consumo de determinados fármacos, como a carbamazepina, fenofibrato, barbitúricos, fenitoína, rifampicina e estrogénios.

\subsubsection{Tratamento}

Perante a identificação de uma lesão primária responsável pelo SC, está recomendada a sua resseção cirúrgica, desde que seja exequível e que previsivelmente reduza o excesso de glicocorticoides. ${ }^{57}$ Em alternativa, poderá ser feita terapêutica com inibidores da esteroidogénese, antagonistas dos recetores dos glicocorticoides ou adrenalectomia bilateral. ${ }^{57}$
O tratamento deve ser adjuvado com anti-hipertensores para o controlo da hipertensão, sendo os IECA/ /ARA a primeira opção. ${ }^{52,57-58}$

\subsection{Feocromocitoma e paraganglioma}

O feocromocitoma e o paraganglioma (FP) são tumores com origem nas células cromafins que, se funcionantes, podem resultar num excesso de libertação 
de catecolaminas no organismo. ${ }^{59} \mathrm{~A}$ maioria destes tumores são feocromocitomas (80 a 85\%), tumores localizados na glândula suprarrenal, ao passo que os paragangliomas são extra-adrenais, com origem nos gânglios do sistema nervoso autónomo. ${ }^{60}$

Os FP são encontrados mais comummente na quarta década de vida e manifestam-se frequentemente com hipertensão, ${ }^{51}$ estimando-se que sejam responsáveis por $0,2 \%$ a $0,6 \%$ dos casos de hipertensão ${ }^{44}$ e por até $1 \%$ dos casos de HR. ${ }^{2}$

\subsubsection{Fisiopatologia}

O excesso de produção e libertação de catecolaminas é o principal mecanismo responsável pela hipertensão associada aos $\mathrm{FP}^{44,59,61}$ uma vez que este se associa a vasoconstrição periférica, aumento do débito cardíaco e ativação do SRAA. ${ }^{61}$

O padrão, quantidade e tipo de catecolaminas libertadas são variáveis, com consequente variabilidade no perfil tensional dos utentes com FP. ${ }^{44,59,61}$

\subsubsection{Manifestações clínicas e/ou laboratoriais}

Os FP manifestam-se classicamente com uma hipertensão paroxística ou sustentada, associada a taquicardia, cefaleias, hipersudorese e palidez. ${ }^{45}$

Utentes com história familiar de FP ou de um síndroma genético com possível associação, presença de uma massa na glândula suprarrenal com características sugestivas, crises hipertensivas despoletadas pela mudança de posição, exercício, procedimentos médicos ou toma de determinados fármacos (como antidepressivos, $\beta$ bloqueadores, analgésicos, metoclopramida, agentes de contraste ou simpaticomiméticos), ou uma diabetes de início recente num jovem magro e hipertenso, devem também levantar a suspeita da existência de FP. ${ }^{44-45,59}$

\subsubsection{Diagnóstico}

De acordo com as guidelines da Endocrine Society, ${ }^{60}$ os testes de primeira linha para a pesquisa do FP são as o doseamento de metanefrinas fracionadas no plasma ou na urina de 24 horas, uma vez que apresentam um elevado valor preditivo negativo.

Nos utentes com alto índice de suspeição, nomeadamente os que apresentam história familiar ou pessoal de FP ou de síndromas genéticos predisponentes, ou um incidentaloma com características imagiológicas sugestivas, o doseamento das metanefrinas fracionadas no plasma deve ser preferido. Nos utentes com baixo índice de suspeição, nomeadamente os que apresentam $\mathrm{HR}$, sinais/sintomas de ativação adrenérgica ou incidentaloma com características pouco sugestivas de FP, deve optar-se pelo doseamento das metanefrinas fracionadas na urina de 24 horas, dada a sua maior especificidade neste grupo de utentes. ${ }^{62}$

Determinados fármacos, como antidepressivos tricíclicos, simpaticomiméticos ou antipsicóticos, devem ser suspensos por pelo menos duas a três semanas antes da colheita das análises. ${ }^{45,60,62}$ Fatores como o stress e a colheita na posição vertical também podem contribuir para resultados falsos-positivos. ${ }^{59,62}$

Se for documentada evidência bioquímica de FP devem ser realizados estudos de imagem, sendo a TC abdomino-pélvica com contraste o exame de primeira linha..$^{60}$ Alguns autores sugerem que perante evidência bioquímica de FP deve ser feita a referenciação imediata a um endocrinologista. ${ }^{45}$

\subsubsection{Tratamento}

O tratamento do FP é a exérese cirúrgica do tumor. ${ }^{59-60}$ Para o controlo da hipertensão, a opção preferencial são os bloqueadores $\alpha$, como a fenoxibenzamina. Se a pressão arterial não atingir os valores-alvo pode-se acrescentar um bloqueador $\beta$ ou um bloqueador dos canais de cálcio ${ }^{59-60} \mathrm{O}$ uso de bloqueador $\beta$ na ausência de um bloqueador $\alpha$ não é recomendado. ${ }^{60}$

\subsection{Coartação da aorta}

A coartação da aorta (CA) é uma constrição do lúmen da artéria aorta, geralmente distal à emergência da artéria subclávia esquerda. ${ }^{5}$ Apesar da sua baixa prevalência, descrita em cerca de 0,06 a $0,08 \%$ da população geral, ${ }^{63}$ a CA torna-se uma causa comum de HS quando se considera a população de crianças e adolescentes hipertensos, particularmente do sexo mas-

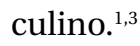

\subsubsection{Fisiopatologia}

A existência de anormalidades estruturais e funcionais da parede arterial, a hipoperfusão renal com subsequente ativação do SRAA e o reajuste do nível de pressão arterial considerada normal pelos barorecetores 
são os mecanismos que contribuem para o desenvolvimento de hipertensão nos utentes com CA. ${ }^{63}$

\subsubsection{Manifestações clínicas e/ou laboratoriais}

A CA é geralmente assintomática, podendo não ser detetada até à idade adulta. ${ }^{3,28,31}$ No entanto, existem sinais sugestivos desta causa, nomeadamente a presença de uma diferença superior a $20 \mathrm{mmHg}$ na pressão arterial sistólica dos membros superiores comparativamente à dos membros inferiores, pulso femoral atrasado ou ausente, ou um sopro sistólico de ejeção com irradiação dorsal..$^{5,10,28}$

Sintomas como cefaleias inexplicáveis, pés frios ou claudicação com o exercício podem ocorrer. A radiografia do tórax pode mostrar a presença de entalhes nas costelas devido ao desenvolvimento de circulação colateral. $^{2-3}$

\subsubsection{Diagnóstico}

O método de diagnóstico de primeira linha para a CA é o ecocardiograma transtorácico; no entanto, é menos preciso no adulto. Em caso de dúvida deve ser realizada uma TC do tórax. ${ }^{64}$

Perante evidência de CA, os utentes devem ser referenciados, consoante a idade, a consulta de cardiologia ou cardiologia pediátrica. ${ }^{65}$

\subsubsection{Tratamento}

O tratamento da CA pode envolver a correção cirúrgica ou a realização de uma angioplastia percutânea com colocação de stent . $^{66}$ No caso de persistência de hipertensão após correção da CA, os bloqueadores $\beta$ podem ser úteis. ${ }^{67}$

\subsection{Fármacos e tóxicos}

O consumo de determinados fármacos ou tóxicos pode ser responsável pelo aparecimento de HS. Exemplos comuns incluem os contracetivos orais, particularmente aqueles com doses altas de estrogénios, anti-inflamatórios não esteroides de uso crónico, antidepressivos (tricíclicos, inibidores seletivos da recaptação de serotonina e inibidores da monoamina oxidase), antipsicóticos atípicos, corticosteroides, descongestionantes nasais, como a fenilefrina e a pseudoefedrina, alguns fármacos para a perda de peso e antiácidos que contêm sódio. ${ }^{1,31}$ Imunossupressores, como a ciclospo- rina ou o tacrolimus, e quimioterápicos, como os inibidores da angiogénese ou da tirosina cinase, também podem causar hipertensão. ${ }^{1,10,31}$

É importante excluir o consumo de álcool, cafeína, anfetaminas, cocaína e esteroides anabolizantes como causas de HS. ${ }^{31}$

\section{Outras causas de hipertensão secundária}

Existem outras causas menos frequentes de HS, como a acromegalia, hiperparatiroidismo primário, tumores da paratiroide, reninoma, síndroma carcinoide, síndroma de Liddle, síndroma de Geller, síndroma de Gordon, síndroma do excesso aparente de mineralo-

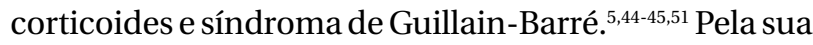
baixa prevalência como causas de HS não serão exploradas nesta revisão.

\section{CONCLUSÃO}

A HS é responsável por cerca de 5 a $10 \%$ dos casos de hipertensão arterial. Em crianças e adolescentes as principais causas de HS são as DPR e a CA. A partir desta idade, as causas mais comuns incluem a SAOS, as DPR, a EAR e o HP.

Avaliar em todos os utentes hipertensos a existência de causas secundárias não é custo-efetivo, pelo que apenas devem ser estudados os utentes com características sugestivas. Posteriormente, o estudo deverá ser dirigido à causa suspeita, quer pelas manifestações clínicas e/ou laboratoriais do utente quer pela prevalência das causas.

O médico de família assume um papel fundamental no reconhecimento de causas secundárias para a hipertensão, uma vez que é frequentemente o primeiro ponto de contacto do utente com o sistema de saúde. Deste modo, este deve ser capaz de solicitar os exames complementares adequados para posterior tratamento ou referenciação, o que assume particular importância dado que quanto mais precocemente forem detetados estes quadros, menor é a probabilidade de se atingir a irreversibilidade das eventuais complicações.

Este trabalho permitiu uma revisão da abordagem das diferentes causas de HS, de acordo com a evidência mais recente. São temas que, pela sua abrangência de patologias abordadas, necessitam de uma revisão constante para adequar a prática do médico de família às práticas mais atuais. 
TABELA 4. Resumo da abordagem da hipertensão secundária nos cuidados de saúde primários

\begin{tabular}{|c|c|c|c|c|}
\hline \multirow{5}{*}{$\begin{array}{l}\text { Principais } \\
\text { causas } \\
\text { de HS }\end{array}$} & & Achados sugestivos & Estudo nos CSP & Referenciação \\
\hline & $\begin{array}{l}\text { Síndroma da apneia } \\
\text { obstrutiva do sono }\end{array}$ & $\begin{array}{l}\text { Roncopatia } \\
\text { Hipersonolência diurna } \\
\text { Aumento da circunferência cervical }\end{array}$ & $\begin{array}{l}\text { Escala de Sonolência de } \\
\text { Epworth } \\
\text { Questionário de Berlim } \\
\text { Polissonografia }\end{array}$ & $\begin{array}{l}\text { Consulta de } \\
\text { medicina do sono }\end{array}$ \\
\hline & $\begin{array}{l}\text { Doenças do } \\
\text { parênquima renal }\end{array}$ & $\begin{array}{l}\text { Alteração do débito urinário } \\
\text { Aumento da creatinina plasmática } \\
\text { Proteinúria } \\
\text { Edemas }\end{array}$ & $\begin{array}{l}\text { Creatinina e ureia plasmática } \\
\text { Análise de urina } \\
\text { Ecografia renal }\end{array}$ & Nefrologia \\
\hline & $\begin{array}{l}\text { Estenose da artéria } \\
\text { renal }\end{array}$ & $\begin{array}{l}\text { Aumento superior a } 50 \% \text { na } \\
\text { creatinina sérica após introdução } \\
\text { de um IECA/ARA } \\
\text { Diferença no tamanho dos rins } \\
\text { superior a } 1,5 \mathrm{~cm} \\
\text { Hipertensão em mulheres de idade } \\
\text { jovem ou em utentes mais velhos } \\
\text { com doença aterosclerótica } \\
\text { conhecida } \\
\text { Sopro abdominal }\end{array}$ & $\begin{array}{l}\text { Ecografia com doppler das } \\
\text { artérias renais }\end{array}$ & Cirurgia vascular \\
\hline & $\begin{array}{l}\text { Hiperaldosteronismo } \\
\text { primário }\end{array}$ & $\begin{array}{l}\text { Hipocalémia inexplicável } \\
\text { Nódulo na glândula suprarrenal } \\
\text { Familiares de } 1 .^{\circ} \text { grau com } \\
\text { hiperaldosteronismo primário }\end{array}$ & $\begin{array}{l}\text { Razão entre a aldosterona e a } \\
\text { atividade da renina } \\
\text { plasmática }\end{array}$ & Endocrinologia \\
\hline \multirow[t]{4}{*}{$\begin{array}{l}\text { Causas } \\
\text { menos } \\
\text { comuns } \\
\text { de HS }\end{array}$} & Disfunção tiroideia & $\begin{array}{l}\text { Hipertiroidismo: palpitações, perda } \\
\text { de peso, intolerância ao calor } \\
\text { Hipotiroidismo: letargia, } \\
\text { intolerância ao frio, aumento de } \\
\text { peso }\end{array}$ & TSH e T4 livre & Endocrinologia \\
\hline & Síndroma de Cushing & $\begin{array}{l}\text { Fácies em «lua cheia», equimoses } \\
\text { fáceis, obesidade abdominal } \\
\text { centrípeta, fraqueza muscular } \\
\text { proximal, estrias roxo-avermelhadas }\end{array}$ & $\begin{array}{l}\text { Cortisol livre na urina de } 24 \\
\text { horas } \\
\text { Teste de supressão noturna } \\
\text { com } 1 \mathrm{mg} \text { de dexametasona }\end{array}$ & Endocrinologia \\
\hline & $\begin{array}{l}\text { Feocromocitoma e } \\
\text { paraganglioma }\end{array}$ & $\begin{array}{l}\text { Hipertensão paroxística } \\
\text { Taquicardia } \\
\text { Cefaleias } \\
\text { Hipersudorese } \\
\text { Palidez }\end{array}$ & $\begin{array}{l}\text { Metanefrinas fracionadas no } \\
\text { plasma ou na urina de } 24 \\
\text { horas } \\
\text { TC abdomino-pélvica com } \\
\text { contraste }\end{array}$ & Endocrinologia \\
\hline & Coartação da aorta & $\begin{array}{l}\text { Diferença superior a } 20 \mathrm{mmHg} \text { na } \\
\text { pressão arterial sistólica dos } \\
\text { membros superiores } \\
\text { comparativamente à dos membros } \\
\text { inferiores } \\
\text { Pulso femoral atrasado ou ausente } \\
\text { Sopro sistólico com irradiação dorsal }\end{array}$ & $\begin{array}{l}\text { Ecocardiograma } \\
\text { TC do tórax }\end{array}$ & $\begin{array}{l}\text { Cardiologia ou } \\
\text { cardiologia } \\
\text { pediátrica }\end{array}$ \\
\hline
\end{tabular}

Legenda: $A R A=$ Antagonistas dos recetores da angiotensina; CSP = Cuidados de saúde primários; HS = Hipertensão secundária; IECA = Inibidores da enzima conversora da angiotensina; TC = Tomografia computorizada. 


\section{REFERÊNCIAS BIBLIOGRÁFICAS}

1. Williams B, Mancia G, Spiering W, Rosei EA, Azizi M, Burnier M, et al. 2018 ESC/ESH Guidelines for the management of arterial hypertension. Eur Heart J. 2018;39(33):3021-104.

2. Rimoldi SF, Scherrer U, Messerli FH. Secondary arterial hypertension: when, who, and how to screen? Eur Heart J. 2014;35(19):1245-54.

3. Charles L, Triscott J, Dobbs B. Secondary hypertension: discovering the underlying cause. Am Fam Med. 2017;96(7):453-61.

4. Taler SJ. Secondary causes of hypertension. Prim Care. 2008;35(3): 489-500.

5. Chiong JR, Aronow WS, Khan IA, Nair CK, Vijayaraghavan K, Dart RA, et al. Secondary hypertension: current diagnosis and treatment. Int J Cardiol. 2008;124(1):6-21.

6. Puar TH, Mok Y, Debajyoti R, Khoo J, How CH, Ng AK. Secondary hypertension in adults. Singapore Med J. 2016;57(5):228-32.

7. Direção-Geral da Saúde. Programa nacional de saúde infantil e juvenil: norma n. ${ }^{\circ}$ 010/2013, de 31/05/2013. Lisboa: DGS; 2013.

8. U.S. Preventive Services Task Force. Screening for primary hypertension in children and adolescents: U.S. Preventive Services Task Force recommendation statement. Ann Intern Med. 2013;159(9):613-9.

9. Whelton PK, Carey RM, Aronow WS, Casey Jr DE, Collins KJ, Himmelfarb CD, et al. 2017 ACC/AHA/AAPA/ABC/ACPM/AGS/APhA/ASH/ ASPC/NMA/PCNA Guideline for the prevention, detection, evaluation, and management of high blood pressure in adults: a report of the American College of Cardiology/American Heart Association Task Force on Clinical Practice Guidelines. J Am Coll Cardiol. 2018;71(19):e127-e248.

10. Textor S. Evaluation of secondary hypertension. UpToDate [Internet]; 2019 [updated $2021 \mathrm{Sep} 8$; cited 2019 Jul 27]. Available from: https:// www.uptodate.com/contents/evaluation-of-secondary-hypertension

11. Chobanian AV, Bakris GL, Black HR, Cushman WC, Green LA, Izzo Jr JL, et al. Seventh report of the Joint National Committee on Prevention, Detection, Evaluation, and Treatment of High Blood Pressure. Hypertension. 2003;42(6):1206-52.

12. Iber C, Ancoli-Israel S, Chesson AL, Quan SF. The AASM manual for the scoring of sleep and associated events: rules, terminology and technical specifications. Darien, IL: American Academy of Sleep Medicine; 2007. p. 15-49.

13. Maspero C, Giannini L, Galbiati G, Rosso G, Farronato G. Obstructive sleep apnea syndrome: a literature review. Minerva Stomatol. 2015; 64(2):97-109.

14. Carey RM, Calhoun DA, Bakris GL, Brook RD, Daugherty SL, DennisonHimmelfarb CR, et al. Resistant hypertension: detection, evaluation, and management: a scientific statement from the American Heart Association. Hypertension. 2018;72(5):e53-e90.

15. Floras JS. Hypertension and sleep apnea. Can J Cardiol. 2015;31(7): 889-97.

16. Gonzaga C, Bertolami A, Bertolami M, Amodeo C, Calhoun D. Obstructive sleep apnea, hypertension and cardiovascular diseases. J Hum Hypertens. 2015;29(12):705-12.

17. DiBona GF. Physiology in perspective: the wisdom of the body. Neural control of the kidney. Am J Physiol Regul Integr Comp Physiol. 2005;289 (3):R633-41.

18. Pedrosa RP, Drager LF, Gonzaga CC, Sousa MG, Paula LK, Amaro AC, et al. Obstructive sleep apnea: the most common secondary cause of hy- pertension associated with resistant hypertension. Hypertension. 2011; 58(5):811-7.

19. Kasai T, Floras JS, Bradley TD. Sleep apnea and cardiovascular disease: a bidirectional relationship. Circulation. 2012;126(12):1495-510.

20. Arzt M, Young T, Finn L, Skatrud JB, Ryan CM, Newton GE, et al. Sleepiness and sleep in patients with both systolic heart failure and obstructive sleep apnea. Arch Intern Med. 2006;166(16):1716-22.

21. Somers VK, White DP, Amin R, Abraham WT, Costa F, Culebras A, et al. Sleep apnea and cardiovascular disease: an American Heart Association/American College of Cardiology Foundation Scientific Statement from the American Heart Association Council for High Blood Pressure Research Professional Education Committee, Council on Clinical Cardiology, Stroke Council, and Council on Cardiovascular Nursing. Circulation. 2008;118(10):1080-111.

22. Direção-Geral da Saúde. Cuidados respiratórios domiciliários - Prescrição de ventiloterapia e outros equipamentos: norma n. ${ }^{\circ}$ 022/2011, de 28/09/2011, atualizada em 11/09/2015. Lisboa: DGS; 2015.

23. Townsend RR. Definition, risk factors, and evaluation of resistant hypertension. UpToDate [Internet]; 2019 [updated 2020 Apr 13; cited 2019 Sep 1]. Available from: https://www.uptodate.com/contents/definition-risk-factors-and-evaluation-of-resistant-hypertension

24. Levey AS, Atkins R, Coresh J, Cohen EP, Collins AJ, Eckardt KU, et al. Chronic kidney disease as a global public health problem: approaches and initiatives - a position statement from Kidney Disease Improving Global Outcomes. Kidney Int. 2007;72(3):247-59.

25. Grams ME, Sang Y, Levey AS, Matsushita K, Ballew S, Chang AR, et al. Kidney-failure risk projection for the living kidney-donor candidate. $\mathrm{N}$ Engl J Med. 2016;374(5):411-21.

26. Warady BA, Srivastava T, Weidemann DK. Chronic kidney disease in children: clinical manifestations and evaluation. UpToDate [Internet]; 2019 [updated 2021 Dec 2; cited 2019 Sep 04]. Available from: https://www. uptodate.com/contents/chronic-kidney-disease-in-children-clinicalmanifestations-and-evaluation

27. Tönshoff B, Kiepe D, Ciarmatori S. Growth hormone/insulin-like growth factor system in children with chronic renal failure. Pediatr Nephrol. 2005;20(3):279-89.

28. Pullalarevu R,Akbar G, Teehan G. Secondary hypertension, issues in diagnosis and treatment. Prim Care. 2014;41(4):749-64.

29. Rosenberg M. Overview of the management of chronic kidney disease in adults. UpToDate [Internet]; 2019 [updated 2021 Nov 29; cited 2019 Sep 4]. Available from: https://www.uptodate.com/contents/ overview-of-the-management-of-chronic-kidney-disease-in-adults

30. James PA, Oparil S, Carter BL, Cushman WC, Dennison-Himmelfarb C, Handler J, et al. 2014 Evidenced-based guideline for the management of high blood pressure in adults: report from the panel members are pointed to the Eighth Joint National Committee (JNC 8). JAMA. 2014; 311(5):507-20.

31. Basile J, Bloch MJ. Overview of hypertension in adults. UpToDate [Internet]; 2019 [updated 2021 Aug 4; cited 2019 Aug 10]. Available from: https://www.uptodate.com/contents/overview-of-hypertension-inadults

32. Textor SC, Lerman L. Renovascular hypertension and ischemic nephropathy. Am J Hypertens. 2010;23(11):1159-69.

33. Fava C, Minuz P, Patrignani P, Morganti A. Renal artery stenosis and ac- 
celerated atherosclerosis: which comes first? J Hypertens. 2006;24(9): 1687-96.

34. Alyamani M, Thomas J, Shanks M, Oudit GY. Resistant hypertension from renal artery stenosis leading to heart failure with preserved ejection fraction. J Investig Med High Impact Case Rep. 2018;6: 2324709618816501.

35. Williams GJ, Macaskill P, Chan SF, Karplus TE, Yung W, Hodson EM, et al. Comparative accuracy of renal duplex sonographic parameters in the diagnosis of renal artery stenosis: paired and unpaired analysis. AJR Am J Roentgenol. 2007;188(3):798-811.

36. Radermacher J, Haller H. The right diagnostic work-up: investigating renal and renovascular disorders. J Hypertens. 2003;21 Suppl 2:S19-S24.

37. Evans KL, Tuttle KR, Folt DA, Dawson T, Haller ST, Brewster PS, et al. Use of renin-angiotensin inhibitors in people with renal artery stenosis. Clin J Am Soc Nephrol. 2014;9(7):1199-206.

38. Ritchie J, Green D, Chrysochou C, Chalmers N, Foley RN, Kalra PA. High-risk clinical presentations in atherosclerotic renovascular disease: prognosis and response to renal artery revascularization. Am J Kidney Dis. 2014;63(2):186-97.

39. Trinquart L, Mounier-Vehier C, Sapoval M, Gagnon N, Plouin PF. Efficacy of revascularization for renal artery stenosis caused by fibromuscular dysplasia: a systematic review and meta-analysis. Hypertension. 2010;56(3):525-32.

40. Funder JW, Carey RM, Mantero F, Murad MH, Reincke M, Shibata H, et al. The management of primary aldosteronism: case detection, diagnosis, and treatment: an Endocrine Society clinical practice guideline. J Clin Endocrinol Metab. 2016;101(5):1889-916.

41. Lee FT, Elaraj D. Evaluation and management of primary hyperaldosteronism. Surg Clin North Am. 2019;99(4):731-45.

42. Kline GA, Prebtani AP, Leung AA, Schiffrin EL. Primary aldosteronism: a common cause of resistant hypertension. Can Med Assoc J. 2017;189 (22):E773-8.

43. Monticone S, Burrello J, Tizzani D, Bertello C, Viola A, Buffolo F, et al. Prevalence and clinical manifestations of primary aldosteronism encountered in primary care practice. J Am Coll Cardiol. 2017;69(14):1811-20.

44. Pappachan JM, Buch HN. Endocrine hypertension: a practical approach. Adv Exp Med Biol. 2017;956:215-37.

45. Yang J, Shen J, Fuller PJ. Diagnosing endocrine hypertension: a practical approach. Nephrology. 2017;22(9):663-77.

46. Berta E, Lengyel I, Halmi S, Zrińyi M, Erdei A, Harangi M, et al. Hypertension in thyroid disorders. Front Endocrinol. 2019;10:482.

47. Prisant LM, Gujral JS, Mulloy AL. Hyperthyroidism: a secondary cause of isolated systolic hypertension. J Clin Hypertens. 2006;8(8):596-9.

48. Beastall GH, Beckett GJ, Franklyn J, Fraser WD, Hickey J, John R, et al. UK guidelines for the use of thyroid function tests [Internet]. London: British Thyroid Foundation; 2006. Available from: https://www.britishthyroid-association.org/sandbox/bta2016/uk_guidelines_for_the_use_ of_thyroid_function_tests.pdf

49. Rossi GP, Barisa M, Allolio B, Auchus RJ, Amar L, Cohen D, et al. The Adrenal Vein Sampling International Study (AVIS) for identifying the major subtypes of primary aldosteronism. J Clin Endocrinol Metab. 2012;97(5):1606-14.

50. Pivonello R, Isidori AM, De Martino MC, Newell-Price J, Biller BM, Colao A. Complications of Cushing's syndrome: state of the art. Lancet Diabetes Endocrinol. 2016;4(7):611-29.
51. Buonacera A, Stancanelli B, Malatino L. Endocrine tumors causing arterial hypertension: pathophysiological mechanisms and clinical implications. High Blood Press Cardiovasc Prev. 2017;24(3):217-29.

52. Barbot M, Ceccato F, Scaroni C. The pathophysiology and treatment of hypertension in patients with Cushing's syndrome. Front Endocrinol. 2019;10:321.

53. Alexandraki KI, Kaltsas GA, Isidori AM, Akker SA, Drake WM, Chew SL, et al. The prevalence and characteristic features of cyclicity and variability in Cushing's disease. Eur J Endocrinol. 2009;160(6):1011-8.

54. Lacroix A, Feelders RA, Stratakis CA, Nieman LK. Cushing's syndrome. Lancet. 2015;386(9996):913-27.

55. Nieman LK, Biller BM, Findling JW, Newell-Price J, Savage MO, Stewart PM, et al. The diagnosis of Cushing's syndrome: an Endocrine Society Clinical Practice Guideline. J Clin Endocrinol Metab. 2008;93(5): 1526-40.

56. Boscaro M, Arnaldi G. Approach to the patient with possible Cushing's syndrome. J Clin Endocrinol Metab. 2009;94(9):3121-31.

57. Nieman LK, Biller BM, Findling JW, Murad MH, Newell-Price J, Savage MO, et al. Treatment of Cushing's syndrome: an Endocrine Society Clinical Practice Guideline. J Clin Endocrinol Metab. 2015;100(8):2807-31.

58. Isidori AM, Graziadio C, Paragliola RM, Cozzolino A, Ambrogio AG, Colao A, et al. The hypertension of Cushing's syndrome: controversies in the pathophysiology and focus on cardiovascular complications. J Hypertens. 2015;33(1):44-60.

59. Pappachan JM, Tun NN, Arunagirinathan G, Sodi R, Hanna FW. Pheochromocytomas and hypertension. Curr Hypertens Rep. 2018;20(1):3.

60. Lenders JW, Duh QY, Eisenhofer G, Gimenez-Roqueplo AP, Grebe SK, Murad MH, et al. Pheochromocytoma and paraganglioma: an Endocrine Society Clinical Practice Guideline. J Clin Endocrinol Metab. 2014; 99(6):1915-42.

61. Canu L, Parenti G, De Filpo G, Mannelli M. Pheochromocytomas and paragangliomas as causes of endocrine hypertension. Front Endocrinol. 2019;10:333.

62. Young JrWF. Clinical presentation and diagnosis of pheochromocytoma. UpToDate [Internet]; 2019 [updated 2021 Apr 13; cited 2019 Sep 15]. Available from: https://www.uptodate.com/contents/clinical-presentation-and-diagnosis-of-pheochromocytoma

63. Law MA, Tivakaran VS. Coarctation of the aorta [Internet]. Treasure Island: StatPearls Publishing; 2021 [updated 2021 Aug 11]. Available from: https://www.ncbi.nlm.nih.gov/books/NBK430913/

64. Vigneswaran TV, Sinha MD, Valverde I, Simpson JM, Charakida M. Hypertension in coarctation of the aorta: challenges in diagnosis in children. Pediatr Cardiol. 2018;39(1):1-10.

65. Pemberton J, Sahn DJ. Imaging of the aorta. Int J Cardiol. 2004;97 Suppl 1:53-60.

66. Bacha E, Hijazi ZM. Management of coarctation of the aorta. UpToDate [Internet]; 2019 [updated 2020 Nov 13; cited 2019 Sep 21]. Available from: https://www.uptodate.com/contents/management-ofcoarctation-of-the-aorta

67. Weber HS, Cyran SE. Endovascular stenting for native coarctation of the aorta is an effective alternative to surgical intervention in older children. Congenit Heart Dis. 2008;3(1):54-9.

68. Moltzer E, Raso FU, Karamermer Y, Boersma E, Webb GD, Simoons ML, et al. Comparison of candesartan versus metoprolol for treatment of 
systemic hypertension after repaired aortic coarctation. Am J Cardiol. 2010;105(2):217-22.

\section{CONFLITO DE INTERESSES}

Os autores declaram não possuir quaisquer conflitos de interesse.

\section{FINANCIAMENTO}

Os autores declaram não existir qualquer financiamento para a presente revisão.

\section{ENDEREÇO PARA CORRESPONDÊNCIA}

Tiago Francisco da Cunha Costa

E-mail: tiago_costa_25@hotmail.com

https://orcid.org/0000-0003-1110-1571

Recebido em 22-10-2019

Aceite para publicação em 26-10-2021

\section{ABSTRACT}

\section{SECONDARY HYPERTENSION: PRIMARY HEALTH CARE APPROACH}

Objectives: Identify situations suggestive of secondary hypertension (SH). Review the pathophysiology, clinical and/or laboratory manifestations, diagnosis, and treatment of the main forms of secondary hypertension as well as establish the role of the family doctor in its approach based on the most recent evidence.

Data sources: MEDLINE/PubMed, The Cochrane Library, UpToDate, U.S. Preventive Services Task Force, and guidelines from Direção-Geral da Saúde.

Methods: Scientific articles were searched on evidence-based medicine online platforms using the keywords 'Secondary Hypertension' and 'Primary Health Care'. The research was conducted in July 2019 and the selection of articles was based on the title, abstract, and publication date. Clinical guidelines were also consulted.

Results: SH should be suspected in situations of resistant hypertension, onset of hypertension before age 30 in a patient without risk factors, sudden increase or instability in basal blood pressure, malignant or accelerated hypertension, the onset of diastolic hypertension in the elderly, hypertension associated with hydro electrolytic disorders or hypertension with clinical features suggestive of a specific etiology. In children and adolescents, the main causes of SH are renal parenchymal diseases, whereas in adults the common causes are obstructive sleep apnea syndrome, renal parenchymal diseases, renal artery stenosis, primary hyperaldosteronism, and pheochromocytoma. Recognition of clinical and/or laboratory manifestations suggestive of the etiology of SH should guide screening tests for further treatment or referral.

Conclusion: Evaluating secondary causes in all hypertensive patients is not cost-effective, so only patients with characteristics suggestive of SH should be studied. Properly performing and interpreting screening tests is critical for the referral and treatment of patients with $\mathrm{SH}$.

Keywords: Secondary hypertension; Primary health care; Obstructive sleep apnea; Renal parenchymal diseases; Renovascular hypertension; Primary hyperaldosteronism. 